Psychother. Psychosom. 1982;38:1-5

\title{
Contents, Vol. 38, 1982
}

\section{Contents}

Dedication 6

Preface 7

Adam J. Krakowski, MD. The Fifth President of the InternationalCollege of Psychosomatic

MedicineKimball, C.P 9

Presidential Papers

Stress and the Practice of Medicine. II. Stressors, Stresses, and StrainsKrakowski, AJ

On Freedom and Induction

Bastiaans, J 24

The Concepts of Specificity Revisited

The Concept of Behavioral Specificity: Three Concepts

Kimball, C.P 32

A Reconsideration of Psychological Specificity in PsychosomaticDisordersNemiah, J.C 39 Shortcomings in Specificity Research and Remedial Suggestions, Including the Involvement of Language MarkersGottschalk, L.A 46

Specificity Revisited: Personality Profiles and Behavioral Issues

Hawkins, D.R 54

Specificity Concepts in Japan

Ago, Y.; Sugita, M; Teshima, H.; Nakagawa, T

Stamina: the Thread of Human Life

Thomas, C.B 74

Contents 4

Alexithymia

Alexithymia: a Psychoanalytic Viewpoint

McDougall, J 81

Empirical Evidence of Alexithymia

Rad, M. von; Lolas, F 91

Influence of Alexithymia on Symbolic Function

Demers-Desrosiers, L 103

Clinical Studies and Approaches

Psychopathology and Verbal Expression in Psychosomatic andPsychoneurotic PatientsTaylor, G.; Doody, K 121

A Prospective Epidemiological Study of Psychosomatic andPsychiatric Syndromes in Young AdultsBinder, J.; Dobler-Mikola, A.; Angst, J 128

Depression and Illness Behavior in a General Hospital:a Prevalence StudyFava, GA.; Pilowsky, I.; Pierfederici, A.; Bernardi, M.; Pathak, D 141

The Dream Specimen in Psychosomatic Medicine in the Light ofClinical ObservationsWarnes, $\mathrm{H}$ 154 
Psychophysiological Disorders in Anxious Patients: Hypertensionand HypotensionFontaine, R.;

Boisvert, J 165

Live Events before the Onset of Rheumatoid Arthritis

Baker, G.H.B 173

'Personality' and Anorexia Nervosa - the Phobic Avoidance Stance.Its Origins and Its

SymptomatologyCrisp, A.H.; Bhat, A.V 178

What Does Clinical Research in Psychosomatics Imply?

Meyer, A.-E 201

Transcultural Studies and Sociocultural Influences

International Comparison of the Prevalence of Psychosomatic Disorders in Schizophrenic

Patients

Ramsay, R.A.; Krakowski, A.J.; Rydzynski, Z.; Jarosz, M.; Engelsmann, F.;Ananth, J 206 Integration of the Psychosomatic Approach with Internal Medicine:Development of Concepts and Preliminary Results of Evaluationof Doctor-Patient InteractionKöhle, K.; Gaus, E.; Simons, C; Westphale, C 221

Contents

5

Some Psychosomatic Disorders in Japan in a Cultural Perspective

Ikemi, Y.; Ikemi, A 231

Teaching Psychosomatic Medicine with Special Reference to theBalint Group and the Case

Supervision GroupFreyberger, H.; Besser, L 239

Blood Pressure and Culture. The Contribution and Cross-CulturalComparisons to

PsychosomaticsMurphy, H.B.M 244

Basic Research and Psychosomatic Influences

Social Class and Psychosomatic Illness

Askevold, F 256

Vascular Response Differences in Field Dependency

Silverman, A.J260

Stress, Coping Behavior and Resistance to Disease

Plaut, S.M.; Friedman, S.B 274

P. Psychosomatic Factors in the Process of Cancerogenesis. TheoreticalModels and Empirical

ResultsGrossarth-Maticek

R.; Kanazir

D.T.; Schmidt

P.; Vetter

$\mathrm{H} \quad 284$

Author Index 303 